\title{
The Bearings-only OF-MD Tracking Algorithm Based on Double Observers Jianfei $\mathrm{Ma}^{1,{ }^{1,},}$, Bing Yan $^{1, \text { a }}$ \\ ${ }^{1}$ Naval University of Engineering, Wuhan 430033, China \\ a438922417@qq.com
}

\begin{abstract}
Due to the measurement noise, the accuracy of the double observers' cross-location method is low. Based on the Fisher information matrix of the double observers, the Cramér-Rao low bound (CRLB) of the double-observers bearings-only tracking is figured out, the proposed optimal fit matrix decomposition algorithm(OF-MD) is used to estimate target's position which do uniform linear motion. Finally, the convergence of the algorithm is compared with the CRLB in different noise cases by the means of simulation. With the increasing number of tracking points, the result is closer to the real target track.
\end{abstract}

Keywords: double observers, bearings-only tracking, CRLB, OF-MD, convergence

\section{Introduction}

Target tracking is the process of real-time estimation of the target motion state based on the mathematical calculation method under the condition of obtaining the observation information about the target. The technology which use the tracking information to extract the state information of the target from the observed information, has been widely used in the fields of traffic control, risk assessment and underwater detection, and developing with the diversity of the demand in different fields and the complexity of the target Track application.

Since the information of the azimuth of the single observer is not enough to track the target without priori information about position and velocity. Compared with single observer, double observers can obtain two pieces of azimuth information about the target at one time, so the double observers' cross-location method can be used to locate the target, but its anti-noise ability is very weak, therefore, considering a full use of observation information and motion model OF-MD double observers' localization algorithm.

\section{Cross localization algorithm of double observers}

The schematic diagram of the double observers' measurement is shown in Fig.1, where $S$ is the true position of the target, $S_{v}$ is the observation position of the target, $\left(x_{o 1}, y_{o 1}\right)$ is the coordinates of observer $O_{1},\left(x_{o 2}, y_{o 2}\right)$ is the coordinates of observer $O_{2}, \theta_{1}$ and $\theta_{2}$ respectively are the true azimuth that $S$ is relative to the observer $O_{1}$ and $O_{2}, \theta_{1 v}$ and $\theta_{2 v}$ respectively are the observed azimuth that $S_{v}$ is relative to the observer $O_{1}$ and $O_{2}, L$ is the distance between the two observers, $O$ is the origin of carrier coordinate system and it is located at the midpoint of the connecting line of $\mathrm{O}_{1}$ with $\mathrm{O}_{2}$.

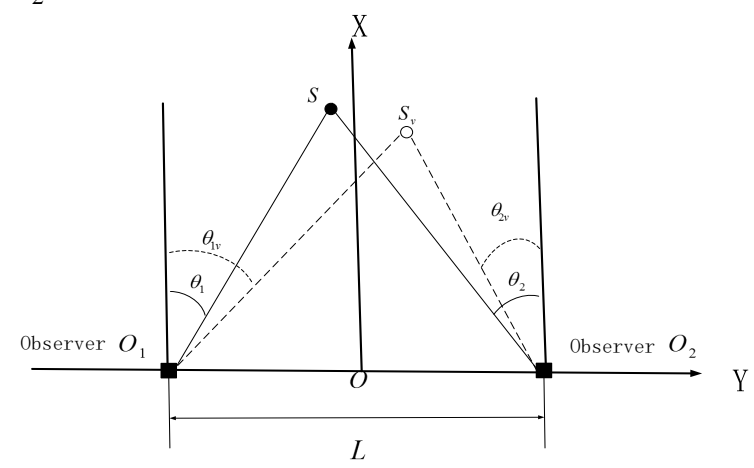

Fig.1 Measurement diagram of double observers

The principle of the cross localization algorithm of double observers is based on the azimuth measured by two observers at the same time on the same target, then figure out the position of the 
target according to the basic the triangulation method[1,2], formula (1) is the solution formula

$$
\left\{\begin{array}{l}
r_{1}=\frac{\cos \theta_{2 v} *\left(y_{o 2}-y_{o 1}\right)+\sin \theta_{2 v} *\left(x_{o 2}-x_{o 1}\right)}{\sin \left(\theta_{1 v}-\theta_{2 v}\right)} \\
x_{1 v}=r_{1} * \cos \theta_{1 v}+x_{o 1} \quad y_{1 v}=r_{1} * \sin \theta_{1 v}+y_{o 1}
\end{array}\right.
$$

\section{Cramér-Rao low bound（CRLB）}

\subsection{CRLB algorithm}

Evaluating the performance of a filter is generally by using simulation or comparing the degree that close to the ideal optimal performance. CRLB as the most popular method for evaluating linear or non-linear estimation of second order error performance can theoretically predict the optimal performance that can be achieved before the system is established. In the time-varying statistical system, the estimated error covariance of any unbiased filter is based on the CRLB matrix [3].

The general nonlinear state formula and observation formula are as follows

$$
\begin{gathered}
\boldsymbol{x}_{k+1}=f_{k}\left(\boldsymbol{x}_{k}\right)+\boldsymbol{\omega}_{k} \\
\mathbf{z}_{k}=h_{k}\left(\boldsymbol{x}_{k}\right)+\boldsymbol{v}_{k}
\end{gathered}
$$

In the formula, state noise $\left\{\boldsymbol{\omega}_{k}\right\}$ and measurement noise $\left\{\boldsymbol{v}_{k}\right\}$ obey the Gaussian distribution that the average is zero, their covariance matrix is $\boldsymbol{Q}_{k}$ and $\boldsymbol{R}_{k}$.

Supposing $\hat{\boldsymbol{x}}_{k}$ is the unbiased estimated value based on the measured values $\boldsymbol{Z}_{k}=\left\{\mathbf{z}_{1}, \mathbf{z}_{2}, \ldots \mathbf{z}_{k}\right\}$, its parameter estimation covariance matrix fits $[4,5]$

$$
\boldsymbol{P}_{k}=E\left(\left(\hat{\boldsymbol{x}}_{k}-x_{k}\right)\left(\hat{\boldsymbol{x}}_{k}-\boldsymbol{x}_{k}\right)^{T}\right) \geq \boldsymbol{J}_{k}^{-1}
$$

In the formula, $\boldsymbol{J}_{k}$ means the Fisher information matrix (FIM). CRLB is defined as the inverse of matrix $\boldsymbol{J}_{k}$.

Marking $\boldsymbol{F}_{k}=\left[\nabla \boldsymbol{x}_{k} f_{k}{ }^{T}\left(\boldsymbol{x}_{k}\right)\right]^{T}$ and $\boldsymbol{H}_{k}=\left[\nabla \boldsymbol{x}_{k} h_{k}{ }^{T}\left(\boldsymbol{x}_{k}\right)\right]^{T}$, they are Jacobian matrix of nonlinear formula $f_{k}$ and $h_{k}$. The recursive formula of FIM information matrix which Tichavsky etal gives is

$$
\boldsymbol{J}_{k+1}=\boldsymbol{Q}_{k}^{-1}-\boldsymbol{Q}_{k}^{-1} E\left(\boldsymbol{F}_{k}\right)\left[\boldsymbol{J}_{k}+E\left(\boldsymbol{F}_{k}^{T} \boldsymbol{Q}_{k}{ }^{-1} \boldsymbol{F}_{k}\right)\right]^{-1} E\left(\boldsymbol{F}_{k}\right) \boldsymbol{Q}_{k}^{-1}+E\left(\boldsymbol{H}_{k+1}{ }^{T} \boldsymbol{R}_{k+1}{ }^{-1} \boldsymbol{H}_{k+1}\right)
$$

When there is no process noise ( $\boldsymbol{Q}_{k}=0$ ), formula (5) can be simplified as

$$
\boldsymbol{J}_{k+1}=\left[\boldsymbol{F}_{k}^{-1}\right]^{T} \boldsymbol{J}_{k} \boldsymbol{F}_{k}^{-1}+E\left\{\boldsymbol{H}_{k+1}{ }^{T} \boldsymbol{R}_{k+1}{ }^{-1} \boldsymbol{H}_{k+1}\right\}
$$

\subsection{CRLB of bearings-only tracking algorithm based on double observers}

In this section, the main point is to compute CRLB for the uniform linear motion target that no process noise. Making the state vector to be estimated is $S=[y(k), x(k), \dot{y}, \dot{x}]^{T}$, where $y(k)$ is the $\mathrm{y}$-axis coordinates, $x(k)$ is the $\mathrm{x}$-axis coordinates, $\dot{y}$ and $\dot{x}$ respectively are the $\mathrm{y}$-axis velocity and the y-axis velocity at time-step $k$. Azimuth sampling cycle is $T$, the vector of azimuth measurements describes as $\boldsymbol{\rho}(k)=\left[\theta_{1 v}(1), \theta_{2 v}(1), \cdots \theta_{1 v}(k), \theta_{2 v}(k)\right]^{T}$, in the vector, $\theta_{i v}(k)$ is the measured azimuth of $k$ moment of $i$ observer [6].

$$
\theta_{i v}(k)=\operatorname{tg}^{-1}\left(\frac{y(k)-y_{o i}}{x(k)-x_{o i}}\right)+\delta \theta_{i}(k)
$$

Defining $\sigma_{1}^{2}$ and $\sigma_{2}^{2}$ respectively as the variance of the measurement error of observers' azimuth, and the variance do not change with time, define $\boldsymbol{R}=\operatorname{diag}\left[\sigma_{1}{ }^{2}, \sigma_{2}{ }^{2}\right]$, then

$$
E\left\{\boldsymbol{H}_{k+1}{ }^{T} \boldsymbol{R}_{k+1}{ }^{-1} \boldsymbol{H}_{k+1}\right\}=\boldsymbol{H}_{k+1}{ }^{T} \boldsymbol{R}^{-1} \boldsymbol{H}_{k+1}
$$

At this time, double observers' bearings-only tracking Fisher information matrix is expressed as 


$$
\boldsymbol{J}_{k}=\left[\boldsymbol{F}_{k-1}^{-1}\right]^{T} \boldsymbol{J}_{k-1} \boldsymbol{F}_{k-1}{ }^{-1}+\boldsymbol{H}_{k+1}{ }^{T} \boldsymbol{R}^{-1} \boldsymbol{H}_{k+1}
$$

Where,

$$
\begin{aligned}
& \boldsymbol{F}_{k}=\left[\begin{array}{llll}
1 & 0 & T & 0 \\
0 & 1 & 0 & T \\
0 & 0 & 1 & 0 \\
0 & 0 & 0 & 1
\end{array}\right] \\
& \boldsymbol{H}_{k}=\frac{\partial \boldsymbol{\rho}(k)}{\partial \boldsymbol{S}}=\left[\begin{array}{cccc}
\frac{x(1)-x_{o 1}(1)}{r_{1}^{2}(1)} & -\frac{y(1)-y_{o 1}(1)}{r_{1}^{2}(1)} & T \frac{x(1)-x_{o 1}(1)}{r_{1}^{2}(1)} & -T \frac{y(1)-y_{o 1}(1)}{r_{1}^{2}(1)} \\
\frac{x(1)-x_{o 2}(1)}{r_{2}^{2}(1)} & -\frac{y(1)-y_{o 2}(1)}{r_{2}^{2}(1)} & T \frac{x(1)-x_{o 2}(1)}{r_{2}^{2}(1)} & -T \frac{y(1)-y_{o 2}(1)}{r_{2}^{2}(1)} \\
\vdots & \vdots & \vdots & \vdots \\
\frac{x(k)-x_{o 1}(k)}{r_{1}^{2}(k)} & -\frac{y(k)-y_{o 1}(k)}{r_{1}^{2}(k)} & T \frac{x(k)-x_{o 1}(k)}{r_{1}^{2}(k)} & -T \frac{y(k)-y_{o 1}(k)}{r_{1}^{2}(k)} \\
\frac{x(k)-x_{o 2}(k)}{r_{2}^{2}(k)} & -\frac{y(k)-y_{o 2}(k)}{r_{2}^{2}(k)} & T \frac{x(k)-x_{o 2}(k)}{r_{2}^{2}(k)} & -T \frac{y(k)-y_{o 2}(k)}{r_{2}^{2}(k)}
\end{array}\right]
\end{aligned}
$$

The corresponding CRLB matrix $\boldsymbol{C}_{k}$ is equal to $\boldsymbol{J}_{k}{ }^{-1}$, so in the case of target do uniform linear motion, double observers' bearings-only CRLB is

$$
\boldsymbol{e}(k)=\sqrt{\boldsymbol{C}_{k}(1,1)+\boldsymbol{C}_{k}(2,2)}
$$

\section{OF-MD bearings-only algorithm of double observers}

As the cross-localization algorithm of double observers doesn't make use of previous observation angles and the information that the target is do uniform linear motion, its anti-noise interference ability is very poor, therefore the optimal fit matrix decomposition algorithm (OF-MD) is proposed to estimate the target's position [6,7].

Making the initial value of the target as its state variable, marked as $\boldsymbol{S}_{c}=\left[y_{c}(0), x_{c}(0), \dot{y}_{c}, \dot{x}_{c}\right]^{T}$, where $y_{c}(0)$ is the initial y-axis coordinates of target, $\dot{x}_{c}$ is the initial x-axis coordinates of target, $\dot{y}_{c}$ and $\dot{x}_{c}$ are the y-direction velocity and x-direction velocity of target, respectively. The observation position is $\boldsymbol{S}_{o i}=\left[y_{o i}, x_{0 i}, 0,0\right]^{T}$. The observation azimuth $\theta_{i v}$ of $i$ observer at time-step $k$ is

$$
\theta_{i v}(k)=\theta_{i}(k)+\delta \theta_{i}(k)
$$

Where $\theta_{i}$ is the true azimuth, $\delta \theta_{i}$ is measuring noise. Through analyzing the movement of the target process, the azimuth formula is expressed as

$$
\theta_{i}(k)=\operatorname{tg}^{-1}\left(\frac{y_{c}(0)+k T \dot{y}_{c}-y_{o i}}{x_{c}(0)+k T \dot{x}_{c}-x_{o i}}\right)
$$

The formula can be further expressed as

$$
\cos \theta_{i}(k)\left[y_{c}(0)+k T \dot{y}_{c}-y_{o i}\right]-\sin \theta_{i}(k)\left[x_{c}(0)+k T \dot{x}_{c}-x_{o i}\right]=0
$$

Substituting formula (13) into the above formula, and simplifying with trigonometric formula, the concluded formula is ${ }^{[8]}$

$$
\begin{gathered}
\cos \theta_{i v}(k)\left[y_{c}(0)+k T \dot{y}_{c}-y_{o i}\right]-\sin \theta_{i v}(k)\left[x_{c}(0)+k T \dot{x}_{c}-x_{o i}\right]=-r_{i}(k) \tan \delta \theta_{i}(k) \\
r_{i}(k)=\sin \theta_{i v}(k)\left[y_{c}(0)+k T \dot{y}_{c}-y_{o i}\right]+\cos \theta_{i v}(k)\left[x_{c}(0)+k T \dot{x}_{c}-x_{o i}\right]
\end{gathered}
$$

In the formula, $r_{i}(k)$ is the radial distance between target's observation positions and observe $O_{i}$. Re-express measurement formula (16) as the form of matrix formula [9] 


$$
\boldsymbol{Z}(k)=\boldsymbol{A}(k) \boldsymbol{S}_{c}+\boldsymbol{V}(k)
$$

In the formula,

$$
\begin{aligned}
& \mathbf{Z}(k)= {\left[\begin{array}{l}
y_{o 1} \cos \theta_{1 v}(1)-x_{o 1} \sin \theta_{1 v}(1) \\
y_{o 2} \cos \theta_{2 v}(1)-x_{o 2} \sin \theta_{2 v}(1) \\
\vdots \\
y_{o 1} \cos \theta_{1 v}(k)-x_{o 1} \sin \theta_{1 v}(k) \\
y_{o 2} \cos \theta_{2 v}(k)-x_{o 2} \sin \theta_{2 v}(k)
\end{array}\right] \quad \boldsymbol{V}(k)=\left[\begin{array}{l}
r_{1}(1) \tan \delta \theta_{1}(1) \\
r_{2}(1) \tan \delta \theta_{2}(1) \\
\vdots \\
r_{1}(k) \tan \delta \theta_{1}(k) \\
r_{2}(k) \tan \delta \theta_{2}(k)
\end{array}\right] } \\
& \mathbf{A}(k)=\left[\begin{array}{cccc}
\cos \theta_{1 v}(1) & -\sin \theta_{1 v}(1) & T \cos \theta_{1 v}(1) & -T \sin \theta_{1 v}(1) \\
\cos \theta_{2 v}(1) & -\sin \theta_{2 v}(1) & T \cos \theta_{2 v}(1) & -T \sin \theta_{2 v}(1) \\
\vdots & \vdots & \vdots & \vdots \\
\cos \theta_{1 v}(k) & -\sin \theta_{1 v}(k) & T \cos \theta_{1 v}(k) & -T \sin \theta_{1 v}(k) \\
\cos \theta_{2 v}(k) & -\sin \theta_{2 v}(k) & T \cos \theta_{2 v}(k) & -T \sin \theta_{2 v}(k)
\end{array}\right]
\end{aligned}
$$

In accordance with the estimated principle of least squares, to find an optimal movement path so that $\sum \delta \theta_{i}^{2}(k)$ is the minimum. Marking $\omega_{i}(k)$ as

$$
\omega_{i}(k)=\left[\sin \theta_{i v}(k), \cos \theta_{i v}(k), k T \sin \theta_{i v}(k), k T \cos \theta_{i v}(k),-\sin \theta_{i v}(k) y_{o i}-\cos \theta_{i v}(k) x_{o i}\right]
$$

So $r_{i}(k)$ can be re-expressed as the formula (20), where the vector $\boldsymbol{\eta}$ is the target motion state vector to be estimated.

$$
\begin{gathered}
r_{i}(k)=\omega_{i}(k) \boldsymbol{\eta} \\
\boldsymbol{\eta}=\left[y_{c}(0), x_{c}(0), \dot{y}_{c}, \dot{x}_{c}, 1\right]^{T}
\end{gathered}
$$

The cost function marked as $J_{a}(k)$ is the sum of squares of two observers' radial distance $r_{i}(k)$ at time-step $k$ and before, which is

$$
J_{a}(k)=\sum_{j=1}^{k} \sum_{i=1,2} r_{i}^{2}(j)=\boldsymbol{\eta}^{T}\left(\sum_{j=1}^{k} \sum_{i=1,2} \boldsymbol{\omega}_{i}^{T}(k) \boldsymbol{\omega}_{i}(k)\right) \boldsymbol{\eta}
$$

Defining $\boldsymbol{W}_{k}$ as

$$
\boldsymbol{W}_{k}=\sum_{j=1}^{k} \sum_{i=1,2} \boldsymbol{\omega}_{i}^{T}(k) \boldsymbol{\omega}_{i}(k)
$$

In addition,

$$
\boldsymbol{V}^{T}(k) \boldsymbol{V}(k)=\boldsymbol{\eta}^{T}[\boldsymbol{A}(k)-\boldsymbol{Z}(k)]^{T}[\boldsymbol{A}(k)-\boldsymbol{Z}(k)] \boldsymbol{\eta}
$$

Defining $\boldsymbol{C}_{k}$ as

$$
\boldsymbol{C}_{k}=[\boldsymbol{A}(k)-\boldsymbol{Z}(k)]^{T}[\boldsymbol{A}(k)-\boldsymbol{Z}(k)]
$$

Consequently, the main idea of state estimation is to find the minimum value of $\lambda$, which meets the formula (26) $[10,11]$

$$
\boldsymbol{\eta}^{T} \boldsymbol{C}_{k} \boldsymbol{\eta}=\lambda J_{a}(k)=\lambda \boldsymbol{\eta}^{T} \boldsymbol{W}_{k} \boldsymbol{\eta}
$$

Since $W_{k}$ is a positive definite matrix, it can be realized that $\lambda$ is the generalized eigenvalue, which $\boldsymbol{C}_{k}$ is relative to $\boldsymbol{W}_{k}$, from the definition of generalized matrix. Marking $\boldsymbol{\eta}_{\min }$ is the generalized eigenvalue of $\lambda_{\min }$. The eigenvector corresponding to the same eigenvalue is not unique (the ratio of any two eigenvectors is $\mu$, which is an arbitrary constant), and there for the vector of the state estimate is 


$$
\hat{\boldsymbol{\eta}}=\frac{1}{\boldsymbol{\eta}_{\min }(5,1)} \boldsymbol{\eta}_{\min }
$$

In conclusion, the final values of the state estimate at time-step $k$ is

$$
\left\{\begin{array}{l}
\hat{y}(k)=\hat{\boldsymbol{\eta}}(1,1)+k T \hat{\boldsymbol{\eta}}(3,1) \\
\hat{x}(k)=\hat{\boldsymbol{\eta}}(2,1)+k T \hat{\boldsymbol{\eta}}(4,1) \\
\hat{\dot{y}}(k)=\hat{\boldsymbol{\eta}}(3,1) \\
\hat{\dot{x}}(k)=\hat{\boldsymbol{\eta}}(4,1)
\end{array}\right.
$$

\section{Simulation analysis}

Target initial point is $(200,-300)$, sailing velocity is $5 \mathrm{~m} / \mathrm{s}$, sailing direction is North East $45^{\circ}$, sailing total time is $200 \mathrm{~s}$, sampling cycle $T$ is $0.5 \mathrm{~s}$, coordinates of observer $O_{1}$ is $(0,-5)$, coordinates of observer $\mathrm{O}_{2}$ is $(0,5)$. At different levels of measured Gaussian noise, the results of Monte-Carlo simulation using the algorithm of cross localization of double observers are shown in Fig.2.

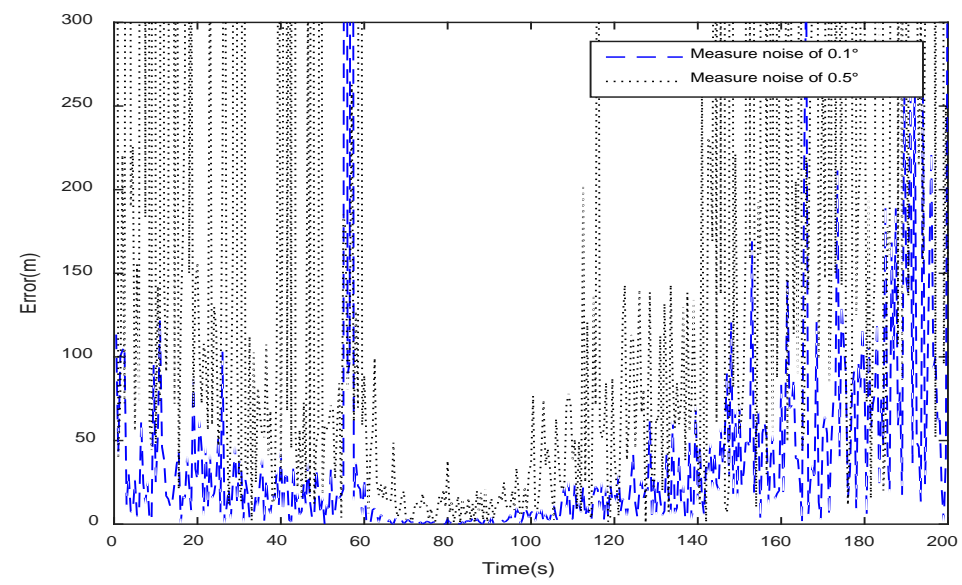

Fig.2 Cross localization error of double observers

In the Fig.2, the localization error of Cross localization of double observers is very large, at the measured Gaussian noise of $0.5^{\circ}$, its localization error even reached the level of 100 meters. In the period of $60 s \sim 100 s$, as the result of the distance between the target and the observer is close, the estimation error of the algorithm is small. Therefore in the case of large measurement noise, the algorithm of cross localization of double observers cannot meet the requirements of tracking targets.

At different levels of measured Gaussian noise, the tracking tracks and tracking errors of OF-MD algorithm are shown in Fig.3a, Fig.3b, Fig.4a, Fig.4b, Fig.5a and Fig.5b. The tracking errors are the result of 20 Monte-Carlo simulations, the tracking track are the result of once simulation. This is due to the fact that the mean error of multiple simulation estimation can be approximated to the true estimation error, and the averaging of multiple simulation estimation results in smoothing of the tracking details. 

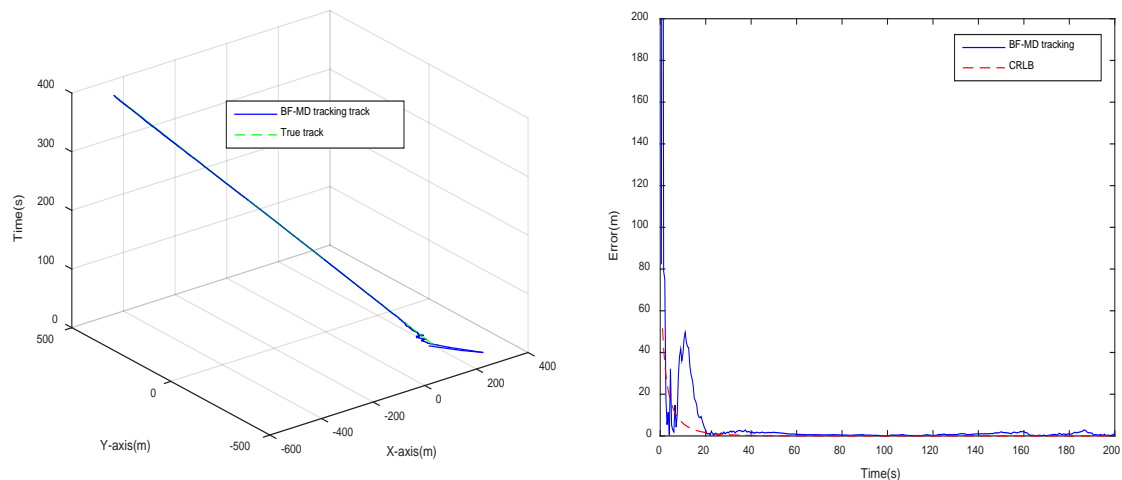

Fig.3a OF-MD tracking curve of $0.1^{\circ}$ noise Fig.3b OF-MD tracking error of $0.1^{\circ}$ noise

As can be seen from Fig. 3a and Fig. 2, at the measured Gaussian noise of $0.1^{\circ}$, the tracking track of the OF-MD algorithm is in good agreement with the actual track, and the tracking error of OF-MD algorithm is much smaller than that of cross localization of double observers, which prove the validity of the algorithm. In the Fig. 3a, after the performance of OF-MD algorithm is stable, its estimation error is less than $3 m$ and converges to the CRLB at $0.1^{\circ}$ measurement noise level, which also indicate the progressive unbiased ability of the algorithm.
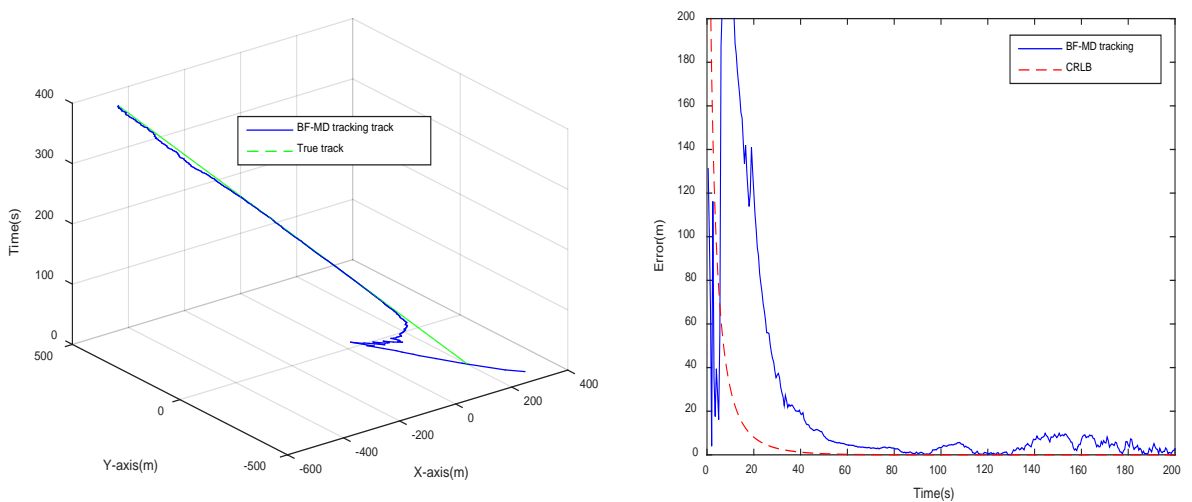

Fig.4a OF-MD tracking curve of $0.5^{\circ}$ noise

Fig.4b OF-MD tracking error of $0.5^{\circ}$ noise
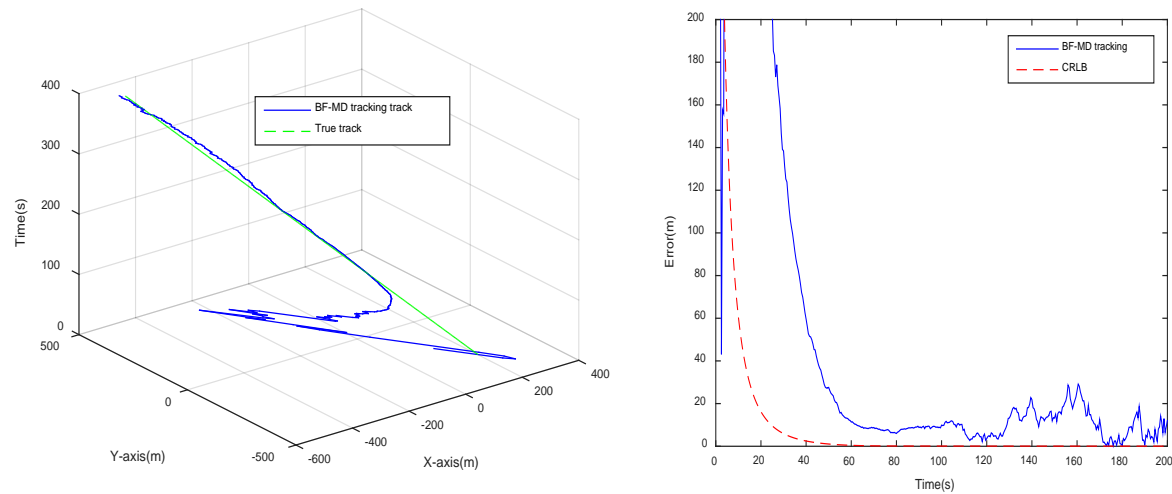

Fig.5a OF-MD tracking curve of $1^{\circ}$ noise

Fig.5b OF-MD tracking error of $1^{\circ}$ noise

As the measurement noise increases, the tracking is still convergent, although the tracking error is rising, and the tracking performance takes longer to stabilize. At the measured Gaussian noise of $0.5^{\circ}$, after the performance of OF-MD algorithm is stable, its estimation error is less than $10 m$ and converges to the CRLB at this measurement noise level. At the measured Gaussian noise of $1^{\circ}$, after the performance of OF-MD algorithm is stable, its estimation error is less than $25 \mathrm{~m}$ and converges to the CRLB at this measurement noise level.

Fig.6a, Fig.6b and Fig.6c are respectively the result of velocity estimation under different levels of noise, it can be seen that the fluctuation of velocity estimation increases with the increase of noise, and the estimation of velocity takes longer to stabilize, but it is generally consistent with the 
true velocity of the target, which also indicate the effectiveness of velocity estimation.

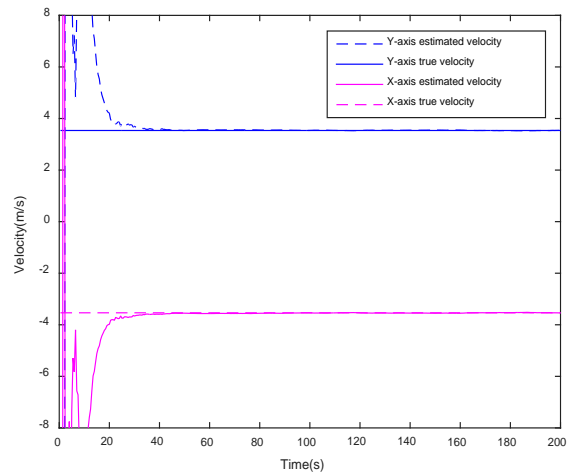

Fig.6a OF-MD estimated velocity of $0.1^{\circ}$ noise

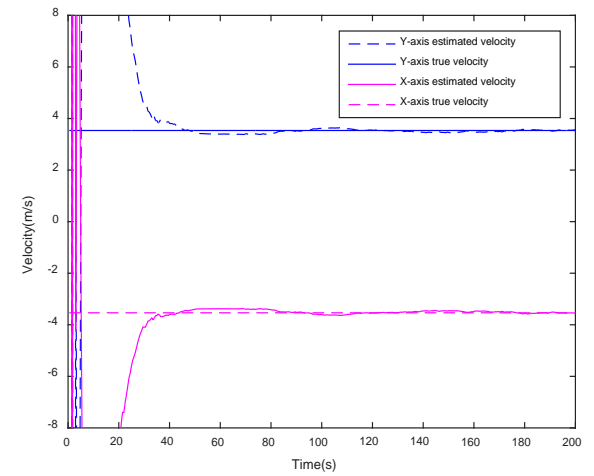

Fig.6b OF-MD estimated velocity of $0.5^{\circ}$ noise

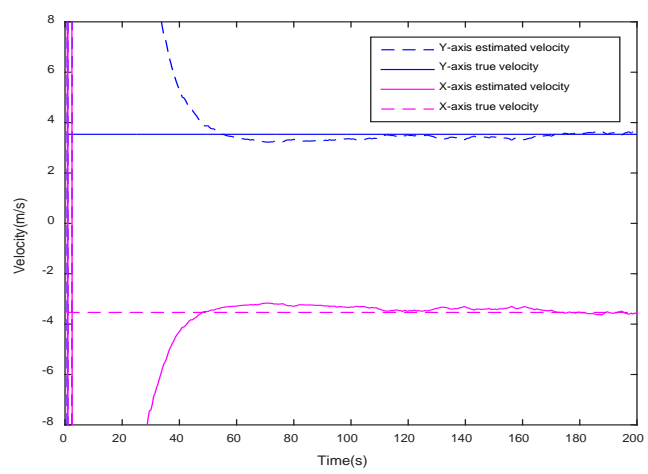

Fig.6c OF-MD estimated velocity of $1^{\circ}$ noise

\section{Conclusion}

The proposed OF-MD bearings-only algorithm of double observers is able to track the uniform linear motion target at a higher level of measurement noise compared to the cross localization algorithm of double observers. OF-MD algorithm is unbiased for the estimation of the target, and converges to the CRLB at same measurement noise level. As the number of observations increases, the estimated track is closer to the real target track. What cannot be ignored is that the estimation error increases at the end of the data, which is not the result of the algorithm, but the result of the distance increases in between the target and observers. Increasing the distance between two observers can effectively reduce such errors.

\section{Acknowledgments}

This work was financially supported by Seismic Detection fund (4010603020402).

\section{References}

[1] FU Sen, KONG Xiang-wei. Bearings-only Target Cross Location of Multi-Station based on Nonlinear Least Squares [J]. Fire Control and Command Control ， 2009，34(8): 80-83.

[2] WANG Yan, YUE Jian-ping. Study on Bearings-only Target Motion Analysis Based on associations of Dual Arrays [J]. ACTA ACUSTICA , 2001, 26(5): 405-409.

[3] NING Jing, LI Yin-ya. A CRLB-based Algorithm for Optimal Observer Maneuvering Trajectory in Bearings-only System[J]. Proceedings of $34^{\text {th }}$ Chinese Control Conference, 2015: 5142-5147. [4] GUO Yun-fei, WEI Wei. Derivation of The CRLB in Nonlinear Filter and Application to Target Tracking [J]. Opto-Electronic Engineering, 2007, 34(4): 27-29.

[5] XU Zhi-gang. State Estimation With Incomplete Measurements and Observer Platform Maneuver Model for TMA [D]. Nanjing University of Science and Technology, Doctoral Thesis, 
2009: 18-25.

[6] XU Ben-lian. The Algorithm Study on Bearings-only Target Localization and Tracking Using Two or More observers [D]. Nanjing University of Science and Technology , Doctoral Thesis, 2006: 17-36.

[7] ZHANG Xiao-feng, ZHAO Jun-wei. Research on Accuracy of Localization Algorithm for Bistatic [J]. JOURNAL OF SYSTEM SIMULATION ， 2001， 26(5): 405-409.

[8] LIU Zhong, DENG Ju-long. Passive Localization Method and Observability Analysis for Multiple Observers Bearings-only System [J]. Fire Control and Command Control ，2004，29(5): 80-83.

[9] NI Zhong-de, FENG Guo-hua. An Algorithm of Multiple-vessel Localization Based on Weighting Least Squares [J]. Transactions of Beijing Institute of Technology ， 2015, 25(11): 971-974.

[10] K.C.Ho, Y.T Chan. An Unbiased Estimator for Bearings-Only Tracking and Doppler-Bearing Tracking[J]. IEEE International Conference on Acoustics， 2003， 28(5): 169-172.

[11] Helferty J P, Mudget D R. Optimal Observer Trajectories for Bearings Only Tracking by Minimizing the trace of Cramer-Rao Lower Bound [C]. Proceedings of $32^{\text {nd }}$ IEEE Conference on Decision and Control, 1993: 936-939. 